\title{
Study on Acoustic Damping Ratio of One Dimensional Sound Field Partitioned with Perforated Plate
}

\author{
Kunihiko Ishihara
}

\begin{abstract}
A perforated plate is widely used as an acoustic absorption material for compressors and acoustic barriers for roads and railways. It has been confirmed that the perforated plate suppressed the self- sustained tone generated from heat exchangers like a boiler by using it on a duct wall. The effects of the perforated plate on the natural acoustic frequency of an one dimensional sound field partitioned with the perforated plate has been studied in our previous investigations. In this paper, the effects of parameters such as an aperture ratio, a hole's length and set positions of the perforated plate in the duct, on the sound pressure level, in other words, acoustic damping are discussed experimentally and analytically. The both results are comparatively agreement. Furthermore it was clarified that the real hole's diameter $d_{00}$ should be used in the response analysis and the equivalent hole's diameter $d_{0}\left(d_{0}=d_{00} \sqrt{ }, n\right.$; Number of holes) should not be used.
\end{abstract}

Index Terms - Noise control, Sound and acoustics, Natural acoustic damping, Perforated plate, Transfer matrix method

\section{INTRODUCTION}

Perforated plates have been used as absorbent materials for a silencer for a compressor and a barrier for roads and rail vehicles [1], [2]. And Ishihara et.al confirmed that the high level sound could be suppressed by applying the perforated plate to the duct wall of the heat exchanger [3], [4] The author has examined the effects of the aperture ratio, the hole length and the plate position on the resonant frequency in an one dimensional acoustic field like a duct [5], [6]. As a result, it was clarified that the resonant frequency decreases with decreasing the aperture ratio and increasing the hole length. And it was also clarified the perforated plate position affects the resonant mode deeply [7].

In this study, I will examine the effects of the aperture ratio, the hole length and the plate position on acoustic damping which is the another acoustic characteristics. On the absorbing action of a single perforated plate, there is the detail study by Melling under the high sound pressure level of $130 \mathrm{~dB}$ [8]. Moreover Utsuno et, al confirmed that the Melling's equation could be applicable under the normal sound pressure level and the amplitude dependency vanished under $80 \mathrm{~dB}[9],[10]$ by comparing the analytical result with the experimental result of absorbent coefficients.

Kunihiko Ishihara, Department of Health and Welfare, Tokushima Bunri University, Shido, Sanuki-city, Kagawa, Japan, +81878997247.
There are many studies to obtain the absorbent coefficients However there are few studies to evaluate the acoustic damping, which is needed when the resonant peak value is desired, Of course, the frequency response can be obtained by analytical method like the Transfer Matrix Method, FEM and BEM with the acoustic impedance. However the acoustic damping ratio is needed in the case of obtaining only the sound pressure level at the resonance. And if the acoustic damping and the sound pressure level at resonance are known once the arbitrary sound pressure level can be obtained immediately like the arbitrary sound pressure =known sound pressure $\times$ (arbitrary acoustic damping/known acoustic damping). That is to say, the obtaining the acoustic damping is very useful.

The sound field is excited by the speaker with the random wave and the frequency responses of the sound pressure level is obtained in the experiment. On the other hand, the frequency response analyses by the Transfer Matrix Method are carried out. The frequency responses have several peaks due to resonance and the half power method is used to obtain the acoustic damping ratio at each mode resonance. In analysis, as the acoustic impedance of the perforated plate is needed Melling's equation is used to calculate the frequency responses.

\section{EXPERIMENTAL APPARATUS AND METHOD}

Figure 1 shows the experimental setup. The perforated plate is inserted at $334 \mathrm{~mm}$ from the left end of the one dimensional duct which has $834 \mathrm{~mm}$ in total length. The experimental parameters are the aperture ratio of the perforated plate and 2 kinds of $1 \%$ and $4 \%$ and the hole length (Plate thickness) are 4 kinds of $2.3 \mathrm{~mm}, 4.6 \mathrm{~mm}, 10$ $\mathrm{mm}$ and $20 \mathrm{~mm}$. One more parameter is the plate set positions which are 3 kinds of (1) $334 \mathrm{~mm}$, (2) $434 \mathrm{~mm}$, (3) $634 \mathrm{~mm}$ from the left end of the duct. These are described as $334 * 500$, $434 * 400,634 * 200$, respectively. Total length is the sum of two numbers and constant. Here the aperture ratio $\varphi$ is defined by $\varphi=\left(\pi \mathrm{d}^{2} / 4\right) \mathrm{N} / \mathrm{S}$. Where the hole diameter is $\mathrm{d}$, the hole number is $\mathrm{N}$ and the duct cross sectional area is $\mathrm{S}$.

\section{ANALYTICAL MODEL AND METHOD}

Figure 2 shows the analytical model. The numbering is performed as shown in Figure 2 and the state vector at each position is described as $\left[P_{\hat{i}}, U_{i \mathfrak{i}}\right]^{T}$. The relation between two 


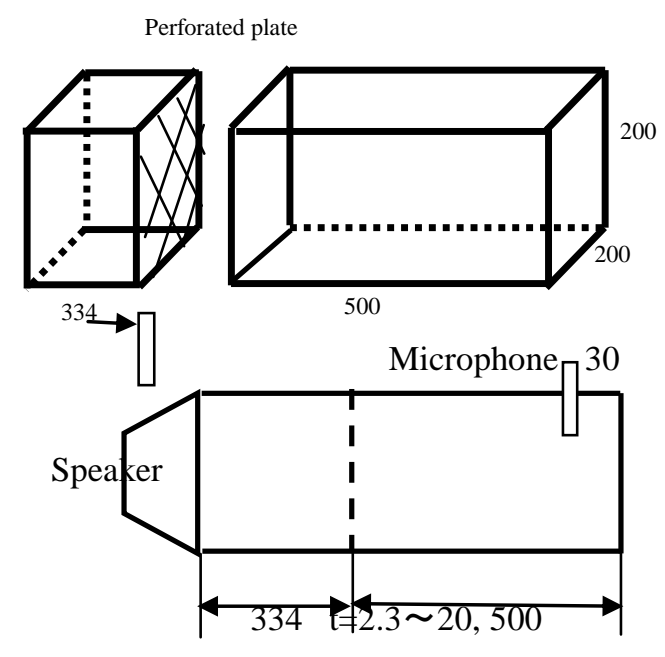

Fig.1 Experimental setup

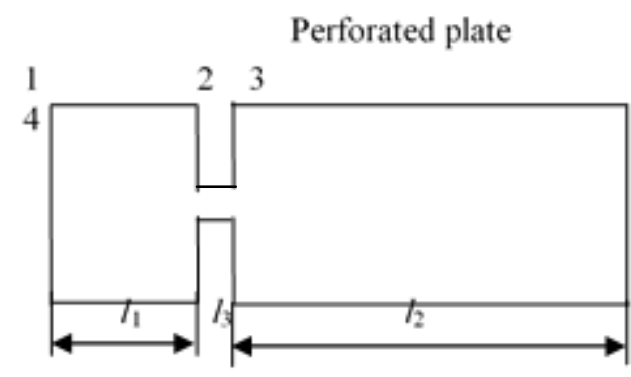

Fig.2 Measuring block diagram

state vectors of both ends can be written by Eq. (1). Where $Z_{\mathrm{i}}$ $=\rho c / S_{\mathrm{i}}, S_{\mathrm{i}}$ is the cross sectional area of each element. And Aij is the result of multiplication of three matrixes. The aperture ratio $\varphi$ is $S_{3} / S_{1}$. Where $S_{3}$ is calculated by $\pi d^{2} / 4 \cdot \mathrm{N}$.

$$
\begin{aligned}
& {\left[\begin{array}{l}
P_{4} \\
\mathrm{U}_{4}
\end{array}\right]=\left[\begin{array}{cc}
\cos k l_{2} & j Z_{2} \sin k l_{2} \\
j \frac{1}{Z_{2}} \sin k l_{2} & \cos k l_{2}
\end{array}\right]\left[\begin{array}{cc}
\cos k l_{3} & j Z_{3} \sin k l_{3} \\
j \frac{1}{z_{2}} \sin k l_{3} & \cos k l_{3}
\end{array}\right] \times} \\
& {\left[\begin{array}{cc}
\cos k l_{1} & j Z_{1} \sin k l_{1} \\
j \frac{1}{Z_{1}} \sin k l_{1} & \cos k l_{1}
\end{array}\right]\left[\begin{array}{l}
P_{1} \\
U_{1}
\end{array}\right]=\left[\begin{array}{ll}
A 11 & A 12 \\
A 21 & A 22
\end{array}\right]\left[\begin{array}{l}
P_{1} \\
U_{1}
\end{array}\right]}
\end{aligned}
$$

The unit forced displacement is applied to the left end of the duct and the right end is closed. So the boundary conditions are given as follows.

$$
U_{1}=1, U_{4}=0
$$

And the relation of state vectors between before and after of the perforated plate is given as follows.

$$
\left[\begin{array}{cc}
1 & Z_{R}+j Z_{i} \\
0 & 1
\end{array}\right] \quad \text { Where } \quad Z=z / S
$$

The acoustic impedance is given by Melling's eq.(4) corresponding to the applicable region $r_{0} \sqrt{\omega \rho / \mu}>10$

$$
\begin{aligned}
& z=\left(2 \mu l_{3} / r_{0}\right) \sqrt{\omega \rho / 2 \mu}(1+j)+j \omega \rho l_{3}=\left(2 \mu l_{a} /\right. \\
& \left.r_{0}\right) \sqrt{\omega \rho / 2 \mu}+j \omega \rho l_{a}\left[1+\frac{1}{\nabla_{0}} \sqrt{\frac{2 \mu}{\omega p}}\right]
\end{aligned}
$$

Where $r_{0}$ is the radius of the hole, $l_{3}$ is the hole length (Plate thickness), $\omega$ is the angular frequency, Air density is $\rho=1.2$ $\mathrm{kg} / \mathrm{m}^{3}$, Air viscosity is $\mu=1.8 \times 10^{-5} \mathrm{~Pa} \cdot \mathrm{s}$.

As a result, A11 A22 of Eq. (1) become the following equations.

$$
\begin{aligned}
& \mathrm{A} 11=\cos k l_{2} \cos k l_{1}-\frac{Z_{\Gamma}}{Z_{\|}} \cos k l_{2} \sin k l_{1} \\
& -\frac{z_{2}}{z_{\|}} \sin k l_{2} \sin k l_{1}+j \frac{z_{k}}{z_{\|}} \cos k l_{2} \sin k l_{1} \\
& \mathrm{~A} 12=-Z_{R} \cos k l_{2} \cos k l_{1} \\
& \text {-j }\left[Z_{1} \cos k l_{2} \sin k l_{1}+Z_{1} \cos k l_{2} \cos k l_{1}+Z_{2} \sin k l_{2} \cos k l_{1}\right] \\
& \begin{aligned}
\mathrm{A} 21= & \frac{Z_{R}}{Z_{1} Z_{2}} \sin k l_{2} \sin k l_{1} \\
& -\mathrm{j}\left[\frac{1}{Z_{2}} \sin k l_{2} \cos k l_{1}-\frac{Z_{P}}{Z_{2} Z_{\|}} \sin k l_{2} \sin k l_{1}+\frac{1}{Z_{1}} \sin k l_{1} \cos k l_{2}\right]
\end{aligned}
\end{aligned}
$$$$
\mathrm{A} 22=\left[\cos k l_{2} \cos k l_{1}-\frac{z_{1}}{Z_{2}} \sin k l_{2} \sin k l_{1}-\frac{z_{P}}{Z_{2}} \sin k l_{2} \cos k l_{1}\right]
$$$$
+j \frac{Z_{R}}{Z_{2}} \sin k l_{2} \cos k l_{1}
$$

The sound pressure of the right end of the duct can be obtained by the Eq. (5)

$$
\text { P4 }=\frac{-A_{11} A_{2}+A_{22} A_{21}}{A_{21}}
$$

The calculations are performed by using the Eq. (5) under the conditions of the aperture ratios of $1 \%$ and $4 \%$, hole lengths of $0.0023 \mathrm{~m}, 0.0046 \mathrm{~m}, 0.01 \mathrm{~m}, 0.02 \mathrm{~m}$. Note, the perforated plate positions are (1) $l_{1}=0.334 \mathrm{~m}, l_{2}=0.5 \mathrm{~m},(2)$ $l_{1}=0.434 \mathrm{~m}, l_{2}=0.4 \mathrm{~m}$, (3) $l_{1}=0.634 \mathrm{~m}, l_{2}=0.2 \mathrm{~m}$. And $r_{0}=0.0015 \mathrm{~m}, \mu=1.882 \times 10^{-5} \mathrm{~Pa} \cdot \mathrm{s}$. The frequency resolution is $10 \mathrm{~Hz}$.

\section{COMPARISON BETWEEN EXPERIMENTAL AND ANALYTICAL RESULTS}

The acoustic damping ratio $\zeta$ can be obtained by Eq. (6). Where $f_{\mathrm{n}}$ is the natural acoustic frequency of $n$ order mode and $f_{1}$ and $f_{2}$ are the frequencies at which the sound pressure value is $1 / \sqrt{ } 2$ of the peak value.

$$
\xi=\frac{f_{2}-f_{1}}{2 f_{2}}
$$

Figure $3 \sim$ Figure 6 show calculation results of the frequency responses for the one dimensional sound field. We will examine the relation between the frequency resolution and the acoustic damping ratio by using these figures. Each frequency resolution is $10.0 \mathrm{~Hz}, 1.0 \mathrm{~Hz}, 0.1 \mathrm{~Hz}$ and $0.01 \mathrm{~Hz}$. As the maximum frequency is $800 \mathrm{~Hz}$, so the calculation points are $80,800,8000,80000$, respectively. The calculation was carried out by using the following values.

$l_{1}=0.634 \mathrm{~m}, l_{2}=0.2 \mathrm{~m}, l_{3}=0.02 \mathrm{~m}, \varphi=0.16, \mathrm{r}_{0}=0.0451 \mathrm{~m}$ $\left(\mathrm{r}_{0}=\sqrt{\mathrm{S} 3 \times \varphi / \pi}\right), \mu=1.882 \times 10^{-5} \mathrm{~Pa} \cdot \mathrm{s}$

Focusing on the first mode peak to examine the relation between the frequency resolution and the peak value, the peak values are $120 \mathrm{~dB}$ at $10 \mathrm{~Hz}, 130 \mathrm{~dB}$ at $1 \mathrm{~Hz}, 140 \mathrm{~dB}$ at $0.1 \mathrm{~Hz}$ 
and $140 \mathrm{~dB}$ at $0.01 \mathrm{~Hz}$ as can be seen from Figure $3 \sim$ Figure 6. And the second and third modes also get the same results as the first mode at $0.1 \mathrm{~Hz}$ and $0.01 \mathrm{~Hz}$. Thereby it can be said that the peak value can be captured correctly for the frequency resolution being less than $0.1 \mathrm{~Hz}$. However we can obtain the acoustic damping by using only Figure 6 in four figures (Figure $3 \sim$ Figure 6 ) and not by using other figures. Because the frequency resolution is coarse in other figures. The frequency resolution can be made small in abundance in calculation but not in experiment. The frequency resolution is determined by giving the maximum frequency and the line number in measurement. So it's often the case that we cannot capture the maximum peak. Then it would have to say that the acoustic damping cannot be obtained correctly in the case of small damping in the experiment.

That is to say, it is presumed easily that the peak value is changed by changing the frequency resolution and the acoustic damping calculated by Eq. (6) is also changed. From this fact, it can be said that it's unlikely that we can obtain the correct acoustic damping ratio in the case of small damping.

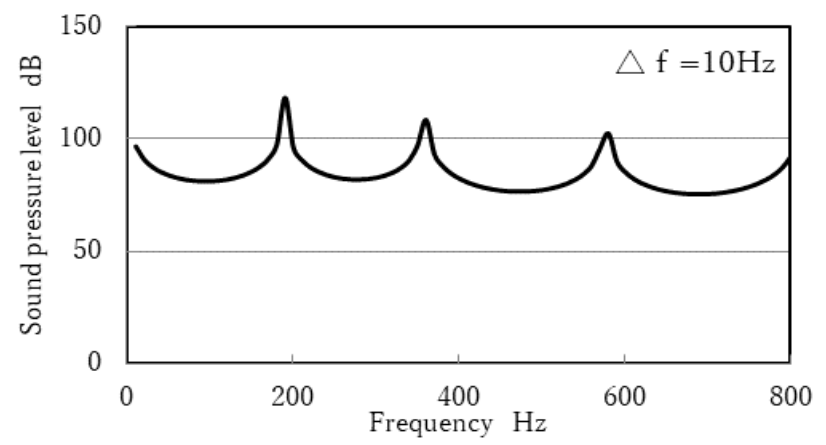

Fig.3 Frequency response of sound pressure level in the case of $\Delta f=10.0 \mathrm{~Hz}$

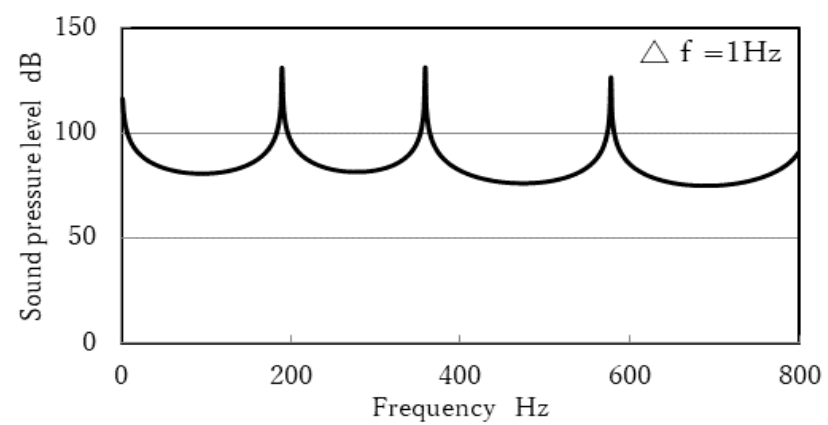

Fig.4 Frequency response of sound pressure level in the case of $\Delta f=1.00 \mathrm{~Hz}$

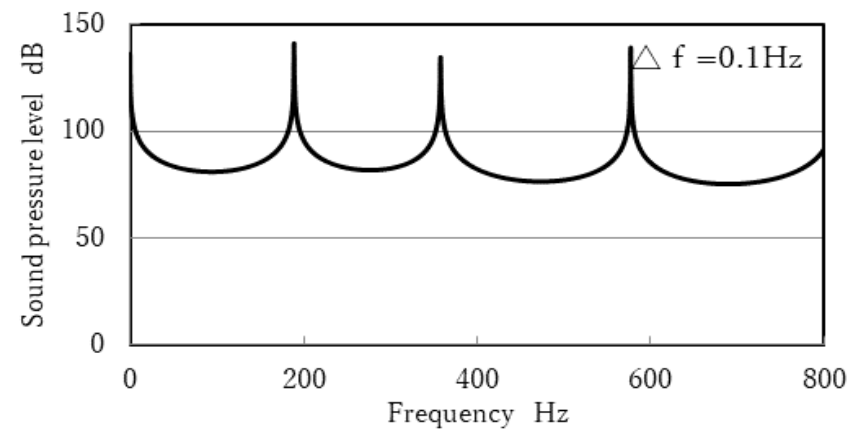

Fig.5 Frequency response of sound pressure level in the case of $\Delta f=0.10 \mathrm{~Hz}$

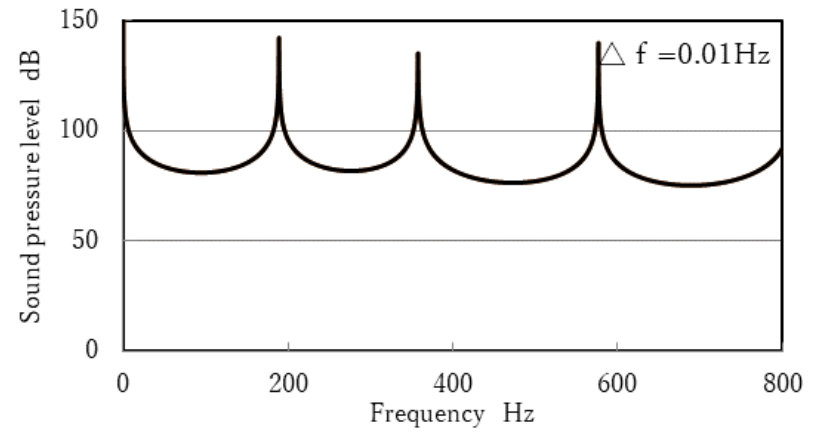

Fig.6 Frequency response of sound pressure level in the case of $\Delta \mathrm{f}=0.01 \mathrm{~Hz}$

Figure 7 shows the results of the frequency responses in the case of $\triangle f=10 \mathrm{~Hz}$ for the initial frequency $f_{0}$ being changed of $2 \mathrm{~Hz}, 4 \mathrm{~Hz}, 6 \mathrm{~Hz}, 8 \mathrm{~Hz}$ and $10 \mathrm{~Hz}$. As can be seen by Figure 7, the peak value of each mode differs depending on the initial frequency $f_{0}$. Then the correct peak value cannot be obtained and also the acoustic damping ratio because of above mentioned. The damping affects in the frequency response only near the resonance. So the absolute value of acoustic damping ratio cannot be obtained correctly unless the peak value can be obtained correctly.

Figure 8 and Figure 9 are calculation results of frequency responses in the cases of $\Delta f=10 \mathrm{~Hz}$ and $\triangle f=0.1 \mathrm{~Hz}$. The notation in figure $\mathrm{ZR} * 10$ shows the calculation result with ten times of ZR (Real part of impedance). Table 1 shows the peak value of each mode of $Z R^{*} 1, Z R * 10, Z R * 100, Z R * 200$ in the case of $\triangle f=10 \mathrm{~Hz}$ and $\triangle f=0.1 \mathrm{~Hz}$

"NAF" in tables shows the natural acoustic frequency. The inverse relation between the amount of damping $\mathrm{ZR}$ and the peak value can be seen in the case of $\triangle f=0.1 \mathrm{~Hz}$. That is to say, it can be seen that when ZR becomes ten times the peak value becomes one tenth and the sound pressure level comes down $20 \mathrm{~dB}$. On the other hand, the inverse relation is not gotten even though the sound pressure level increases with decreasing ZR in the case of $\triangle f=10 \mathrm{~Hz}$. Therefore it can be said that the data processing should be performed with suitable frequency resolution to discuss the damping.

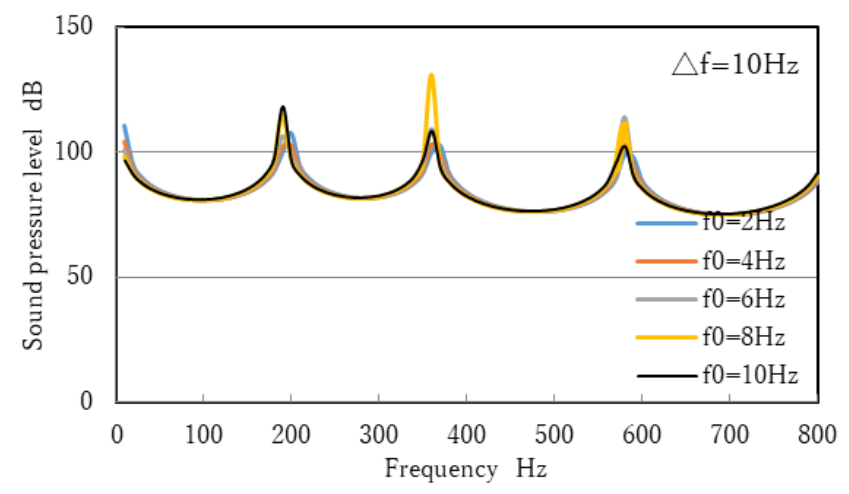

Fig.7 Effect of initial frequency on frequency response of sound pressure level in the case of $\Delta f=10 \mathrm{~Hz}$ 


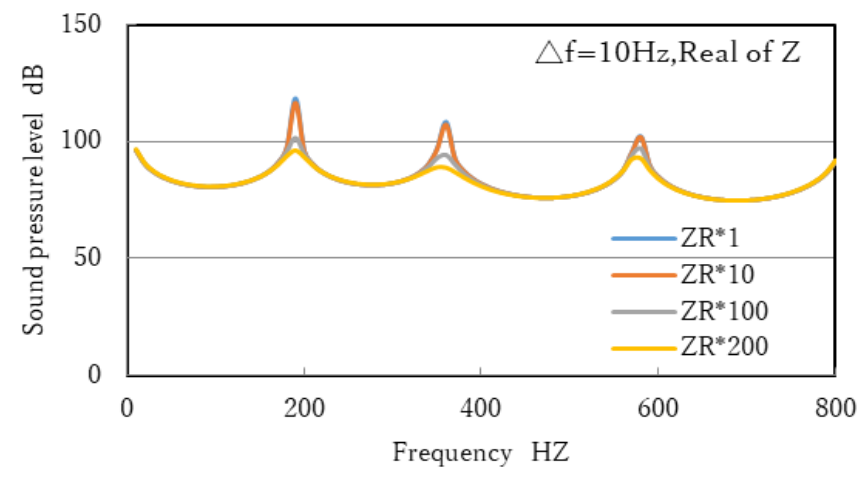

Fig.8 Frequency response of sound pressure level in the case of $\Delta f=10.0 \mathrm{~Hz}$ for various values of $\mathrm{ZR}$

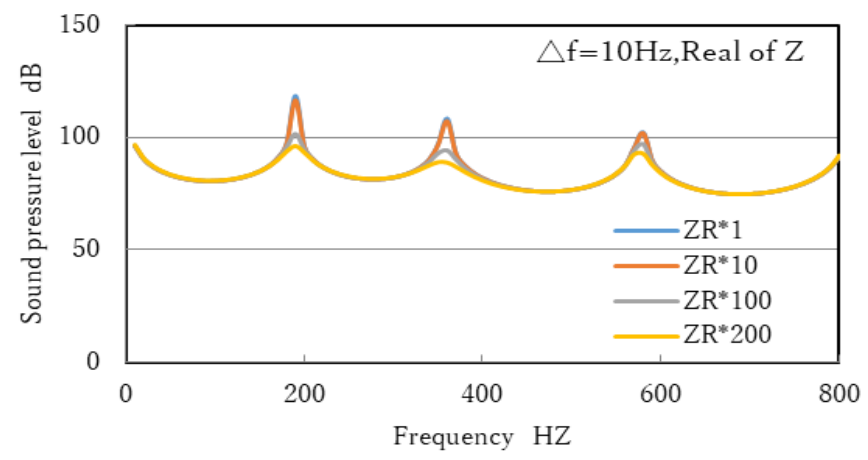

Fig.9 Frequency response of sound pressure level in the case of $\Delta f=0.10 \mathrm{~Hz}$ for various values of $\mathrm{ZR}$

Table 1a: Relation between each peak SPL and variation of real part of impedance $\mathrm{z}$

$\boldsymbol{f = 1 0 H z}$
\begin{tabular}{r|r|r|r|r|} 
N.A.F. & ZR*1 & ZR*10 & $\begin{array}{l}\text { ZR*10 } \\
\mathbf{0}\end{array}$ & $\begin{array}{l}\text { ZR*20 } \\
\mathbf{0}\end{array}$ \\
\hline $\mathbf{1 9 0 H}$ & 118.23 & 116.73 & 101.92 & 95.983 \\
$\mathbf{z}$ & 5 & 3 & 8 & 9 \\
\hline $\mathbf{3 6 0 H}$ & 108.34 & 107.46 & 94.630 & 88.750 \\
$\mathbf{z}$ & & 7 & 9 & 9 \\
\hline $\mathbf{5 8 0 H}$ & 102.22 & 102.14 & 97.396 & 92.604 \\
$\mathbf{z}$ & 8 & 1 & 1 & 6 \\
\hline
\end{tabular}
Difference not 20dB not 20dB not 6dB

Table 1b: Relation between each peak SPL and variation of real part of impedance $\mathrm{z}$

\section{$\Delta f=0.1 \mathrm{~Hz}$}

\begin{tabular}{|l|r|r|r|r|}
\hline N.A.F. & $Z \mathrm{ZR} * 1$ & $Z \mathrm{ZR} * 10$ & $\begin{array}{l}\text { ZR*10 } \\
\mathbf{0}\end{array}$ & $\begin{array}{l}\text { ZR*20 } \\
\mathbf{0}\end{array}$ \\
\hline $\mathbf{1 8 9 . 2 H z}$ & 141.41 & 122.01 & 102.01 & 95.996 \\
& 4 & 1 & 7 & 7 \\
\hline $\mathbf{3 5 7 . 9 H z}$ & 134.87 & 114.98 & 94.980 & 88.960 \\
& 7 & 8 & 2 \\
\hline $\mathbf{5 7 6 . 8 H z}$ & 139.41 & 119.69 & 99.695 & 93.675 \\
& 8 & 3 & 9 & 3 \\
\hline
\end{tabular}

\section{COMPARISON OF ACOUSTIC DAMPING BETWEEN ANALYTICAL AND EXPERIMENTAL VALUES}

Here the comparison of acoustic damping due to the perforated plate between theoretical and experimental values will be discussed. The theoretical value means the acoustic damping ratio itself due to the perforated plate but the experimental value contains the acoustic damping in the case of no perforated plate.

Then the acoustic damping ratio $\zeta$ due to the perforated plate in experiments will be obtained as follows. First the acoustic damping $\zeta_{0}$ in the case of no perforated plate will be obtained and next the acoustic damping $\zeta_{d}$ in the case of with perforated plate will be obtained. And true acoustic damping due to only the perforated plate in the experiment will be calculated by $\zeta=\zeta_{\mathrm{d}}-\zeta_{0}$.

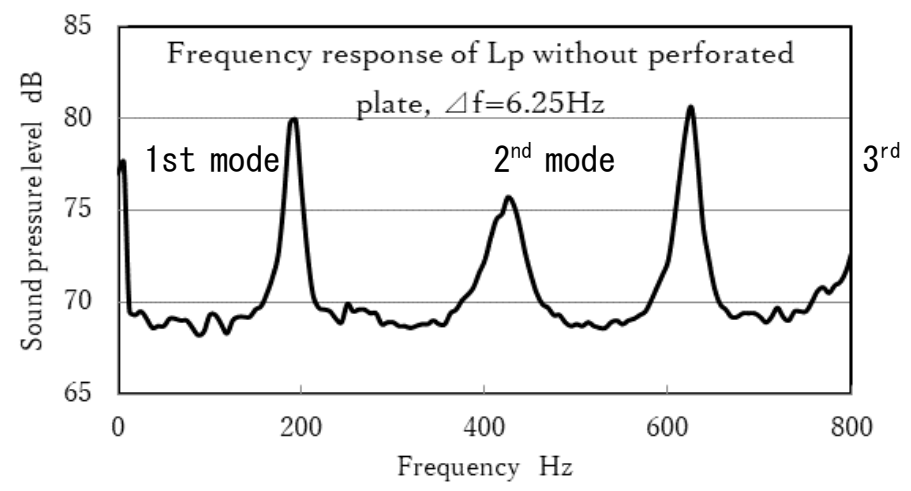

Fig.10 Frequency response of sound pressure level of duct in the case of without perforated plate
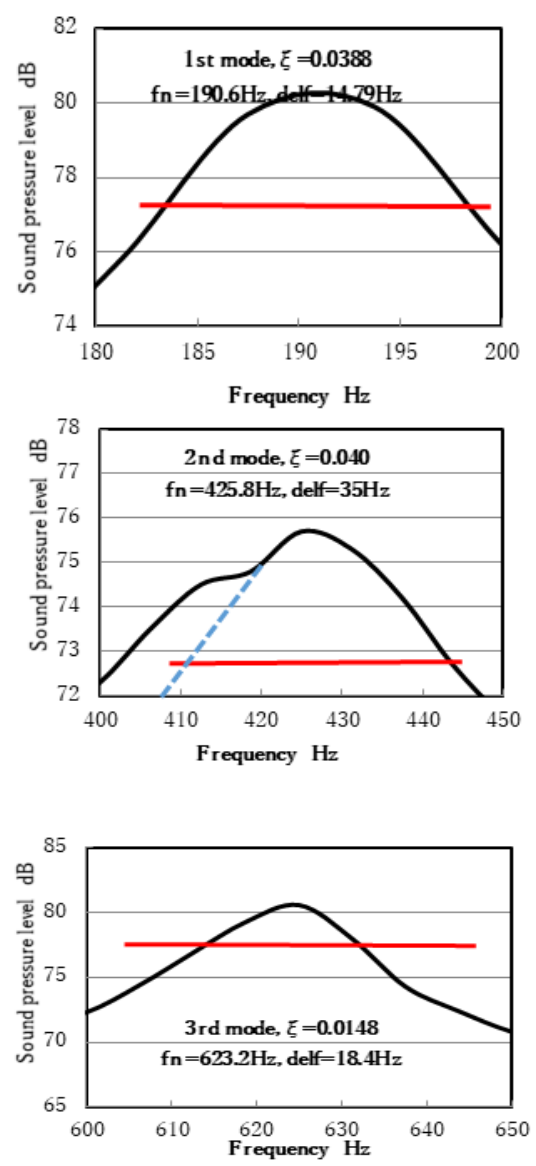

Fig.11 Expanding figures of resonance parts of three modes 
Figure 10 shows the frequency response of the sound pressure level in frequency range $0 \sim 800 \mathrm{~Hz}$ in the duct without the perforated plate. We can see three peaks and they are the first, the second and the third mode peak, respectively. Here the frequency resolution is $6.25 \mathrm{~Hz}$. $\Delta f$ becomes $\Delta$ $f=2 \zeta f_{\mathrm{n}}=4 \sim 40 \mathrm{~Hz}$ because the acoustic damping is about 0.01 $\sim 0.1$.

We cannot obtain the acoustic damping correctly because the frequency resolution $6.25 \mathrm{~Hz}$ is too much course. Then here the resonance part is enlarged as shown in Figure 11 for each mode and $f_{\mathrm{n}}, f_{1}, f_{2}$ are obtained and the acoustic damping is calculated. On the other hand, the acoustic damping can be obtained by the frequency resolution being made very small in analysis.

As a result, the acoustic damping ratio is 0.0388 for first mode, 0.040 for second mode and 0.0148 for third mode in the case of no perforated plate. Another experiment has a result of 0.030 for first mode, 0.030 for second mode and 0.013 for third mode in the case of no perforated plate. Then two experimental results can be said to be about the same by taking into account the difficulty of the evaluation of the damping in the measurement. That is to say, the acoustic damping of the perforated plate itself can be obtained by using these values.

Table 2 Table 4 show acoustic damping ratios of ducts of which the perforated plates are set at $334 \mathrm{~mm}, 434 \mathrm{~mm}$ and $634 \mathrm{~mm}$ from the left ends. These acoustic damping ratios are shown for the first, the second and the third mode by changing hole lengths (plate thickness).

Hatching parts in these tables colored by yellow are values of which the experimental value is comparatively agreement with the analytical values. Here "comparatively agreement" means $0<$ experimental value/analytical value $<1.4$. Values noted in brackets (For example 22/24=0.916 in Table 2) means percent of degree of coincidence. From these results, it can be said that almost all the analytical values coincide with experimental values. That is to say, the acoustic impedance of the perforated plate by Melling could be used to the frequency response analysis.

The following findings can be seen from these tables.

(1) The theoretical and experimental values of the acoustic damping for the first mode become larger with smaller the aperture ratio. However it's tendency cannot be seen for the second and the third modes.

(2) The theoretical acoustic damping becomes larger with becoming the mode order $\mathrm{n}$ smaller.

Table 2: Comparison between experimental and theoretical results of duct $334 * 500$

Red: Experiment, Blue: Calculation $(22 / 24=0.916)$

\begin{tabular}{|r|c|c|c|c|c|c|}
\hline & \multicolumn{2}{|c|}{$\mathbf{1 s t}$} & \multicolumn{2}{c|}{ 2nd } & \multicolumn{2}{c|}{ 3rd } \\
\hline \multirow{2}{*}{} & $\mathbf{1 \%}$ & $\mathbf{4 \%}$ & $\mathbf{1 \%}$ & $\mathbf{4 \%}$ & $\mathbf{1 \%}$ & $\mathbf{4 \%}$ \\
\hline \multirow{2}{*}{$\boldsymbol{l}_{\mathbf{3}}=\mathbf{2 . 3 m m}$} & 0.0309 & 0.0227 & 0.0085 & 0.0137 & 0.0096 & 0.0072 \\
\cline { 2 - 7 } & 0.0352 & 0.0208 & 0.0139 & 0.0165 & 0.0082 & 0.0056 \\
\hline \multirow{2}{*}{$\boldsymbol{l}_{\mathbf{3}} \mathbf{4 . 6 m m}$} & 0.0620 & 0.0563 & 0.0124 & 0.0198 & 0.0107 & 0.0104 \\
\hline \multirow{2}{*}{$\boldsymbol{l}_{\mathbf{3}}=\mathbf{1 0} \mathbf{m m}$} & 0.0406 & 0.0346 & 0.0164 & 0.0136 & 0.0085 & 0.0127 \\
\hline \multirow{2}{*}{$\boldsymbol{l}_{\mathbf{3}}=\mathbf{2 0 m}$} & 0.0840 & 0.0745 & 0.0087 & & 0.0087 & 0.0094 \\
\cline { 2 - 7 } $\mathbf{m}$ & 0.0600 & 0.0663 & 0.0122 & 0.0107 & 0.0088 & 0.0060 \\
\cline { 2 - 7 } & 0.0934 & 0.0691 & 0.0088 & 0.0027 & 0.0106 & 0.0093 \\
\hline
\end{tabular}

Next, the RET (Ratio of Experiment to Theory ) was obtained to compare the theoretical and experimental values These results are shown in Figure 12, Figure 13 and Figure 14. If the experimental value coincides with the analytical value,

Table 3: Comparison between experimental and theoretical results of duct $434 * 400$

Red: Experiment, Blue: Calculation $(21 / 24=0.875)$

\begin{tabular}{|c|c|c|c|c|c|c|}
\hline & \multicolumn{2}{|c|}{ 1st } & \multicolumn{2}{|c|}{ 2nd } & \multicolumn{2}{|c|}{ 3rd } \\
\hline & $1 \%$ & $4 \%$ & $1 \%$ & $4 \%$ & $1 \%$ & $4 \%$ \\
\hline \multirow{2}{*}{$l_{3}=2.3 \mathrm{~mm}$} & 0.0232 & 0.0230 & 0.0132 & 0.0075 & 0.0153 & 0.0078 \\
\hline & 0.0579 & 0.0297 & 0.0161 & 0.0096 & 0.0092 & 0.0082 \\
\hline \multirow{2}{*}{$l_{3}=4.6 \mathrm{~mm}$} & 0.0078 & 0.0318 & 0.0098 & 0.0134 & 0.0146 & 0.0087 \\
\hline & 0.0408 & 0.0251 & 0.0164 & 0.0120 & 0.0184 & 0.0074 \\
\hline \multirow{2}{*}{$l_{3}=10 \mathrm{~mm}$} & 0.1232 & 0.0550 & 0.0051 & 0.0163 & 0.0143 & 0.0160 \\
\hline & 0.0805 & 0.0400 & 0.0066 & 0.0193 & 0.0105 & 0.0122 \\
\hline \multirow{2}{*}{$\begin{array}{r}l_{3}=20 \mathrm{~m} \\
\mathrm{~m}\end{array}$} & 0.1394 & 0.0589 & 0.0085 & 0.0094 & 0.0155 & 0.0153 \\
\hline & 0.0809 & 0.0412 & 0.0099 & 0.0115 & 0.0115 & 0.0101 \\
\hline
\end{tabular}

Table 4: Comparison between experimental and theoretical results of duct $634 * 200$

Red: Experiment, Blue: Calculation $(23 / 24=0.958)$

\begin{tabular}{|c|c|c|c|c|c|c|}
\hline & \multicolumn{2}{|c}{ 1st } & \multicolumn{2}{c|}{ 2nd } & \multicolumn{2}{c|}{ 3rd } \\
\hline \multirow{2}{*}{} & $\mathbf{1 \%}$ & $\mathbf{4 \%}$ & $\mathbf{1 \%}$ & $\mathbf{4 \%}$ & $\mathbf{1 \%}$ & $\mathbf{4 \%}$ \\
\hline \multirow{2}{*}{$\boldsymbol{l}_{\mathbf{3}}=\mathbf{2 . 3 m m}$} & 0.0852 & 0.0334 & 0.0418 & 0.0147 & 0.0039 & 0.0067 \\
\cline { 2 - 7 } & 0.0269 & 0.0409 & 0.0638 & 0.0142 & 0.0053 & 0.0117 \\
\hline \multirow{2}{*}{$\boldsymbol{l}_{\mathbf{3}}=\mathbf{4 . 6 m m}$} & 0.0563 & 0.0526 & 0.0377 & 0.0125 & 0.0041 & 0.0046 \\
\hline \multirow{2}{*}{$\boldsymbol{l}_{\mathbf{3}}=\mathbf{1 0} \mathbf{m m}$} & 0.0443 & 0.0437 & 0.0329 & 0.0123 & 0.0048 & 0.0063 \\
\hline \multirow{2}{*}{$\boldsymbol{l}_{\mathbf{3}}=\mathbf{2 0 m}$} & 0.0555 & 0.0408 & 0.0475 & 0.0226 & 0.0139 & 0.0124 \\
\cline { 2 - 7 } $\mathbf{m}$ & 0.0420 & 0.0524 & 0.0574 & 0.0217 & 0.0092 & 0.0082 \\
\cline { 2 - 7 } & 0.0852 & 0.0334 & 0.0418 & 0.0147 & 0.0039 & 0.0067 \\
\hline
\end{tabular}

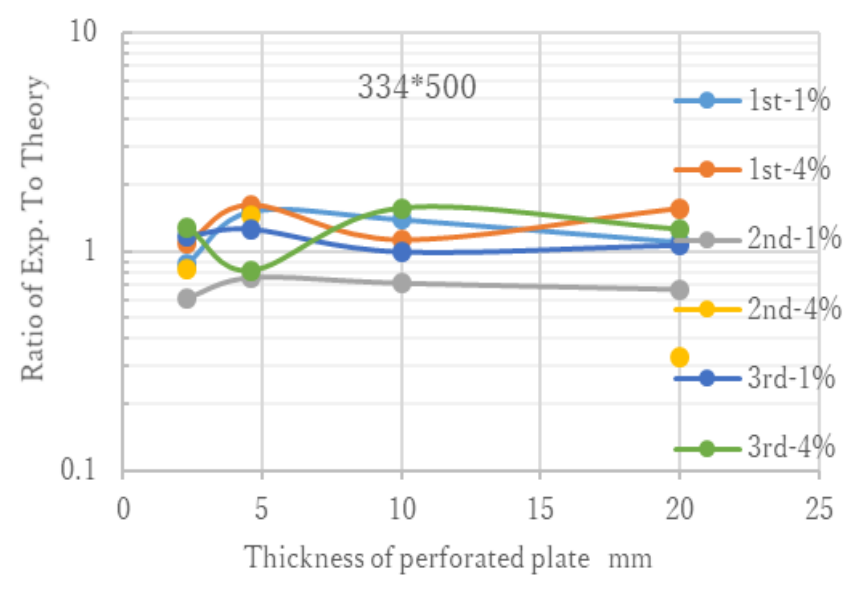

Fig.12 Ratio of experimental value to theoretical value in the case of duct $334 * 500$ 


\section{Study on Acoustic Damping Ratio of One Dimensional Sound Field Partitioned with Perforated Plate}

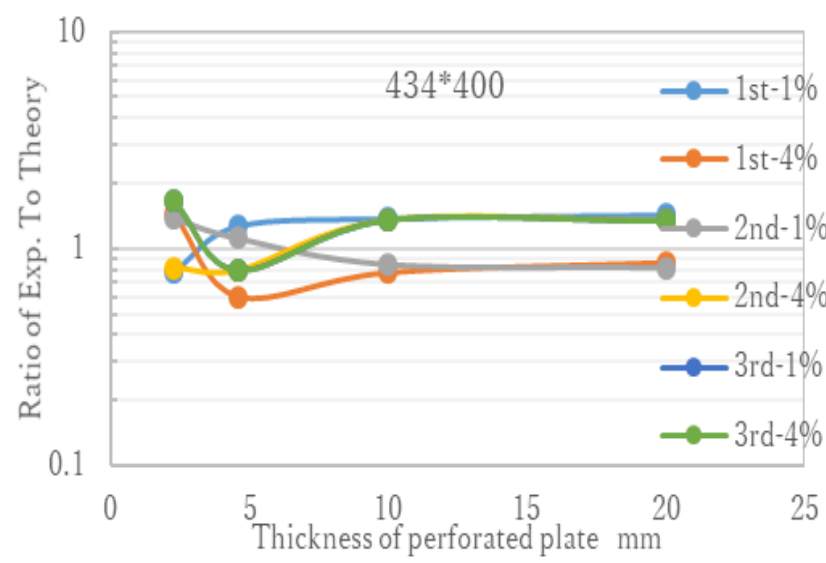

Fig.13 Ratio of experimental value to theoretical value in the case of duct $434 * 400$

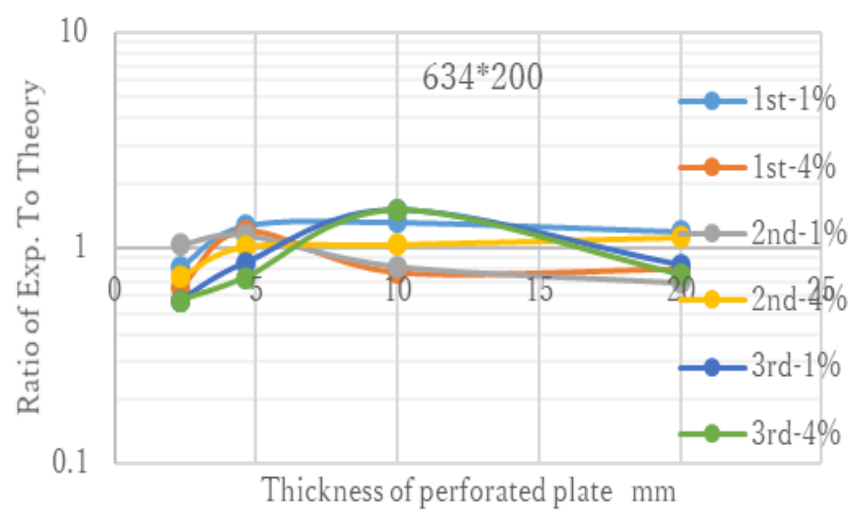

Fig.14 Ratio of experimental value to theoretical value in the case of duct $334 * 500$

the value of RET becomes 1.0. Here it is evaluated to be agree if the RET is larger than 0.7 and less than 1.4. As a result, it can be said that almost all the values are agreement with each other and the theoretical calculation is useful for the evaluation of acoustic damping.

Figure 15 shows the result of the frequency response for two cases of different diameter of holes under the condition of the aperture ratio $1 \%$ and plate thickness $20 \mathrm{~mm}$. One case is for $\mathrm{d}_{00}=3 \mathrm{~mm}$ and hole number $\mathrm{N}$ and the other case is one hole and the diameter $d_{0}$ which is calculated by $d_{00} \times \sqrt{N}$. It can be seen that the peak value of the sound pressure level calculated by using $\mathrm{d}_{00}$ is smaller than the peak by using $\mathrm{d}_{0}$.

This fact is of particular note not only for the first mode but also the second and the third modes become small.

Then, it is important that the real hole diameter $\mathrm{d}_{00}$ should be used in calculation of the real part of impedance. If $d_{0}$ is used the damping is evaluated smaller than the reality. As a result, the peak value is obtained larger than the reality.

On the other hand, the imaginary part of the acoustic impedance is allowed to be used $\mathrm{d}_{0}$ (Equivalent diameter) but the open end correction should be calculated by using $\mathrm{d}_{00}$. Of course $\mathrm{d}_{00}$ can be used in calculation of the natural acoustic frequency.

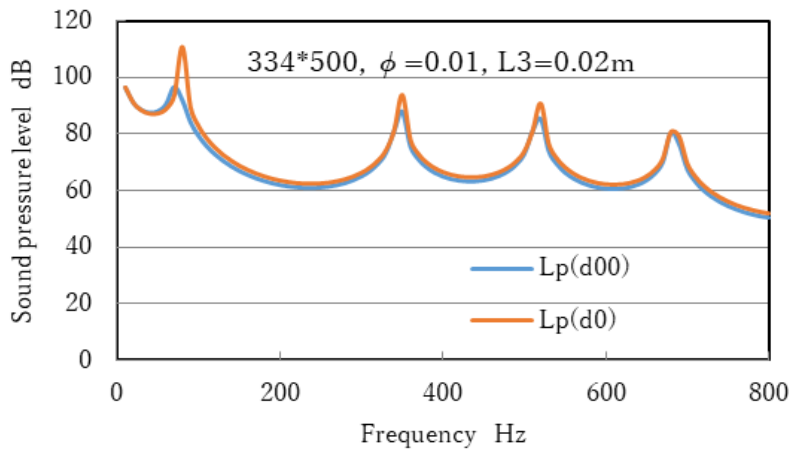

Fig. 15 Comparison of results using $\mathrm{d}_{00}$ and $\mathrm{d}_{0}$ in case of duct $334 * 500$

\section{CONCLUSIONS}

In order to examine effects of the aperture ratio, the hole length and set positions of the perforated plate on the sound pressure level of the duct sound field, the analysis by the transfer matrix method and the experiment are performed and the both results are compared especially in focus the acoustic damping. As a result, the following findings can be obtained.

1) The acoustic damping obtained by the frequency response by using Melling's equation as the impedance of the perforated plate is agreement with one obtained by the experiment.

2) The inverse relation exists between the acoustic damping ratio of the duct and the sound pressure at resonance.

3) When we will try to obtain the small damping the suitable frequency resolution should be selected.

4) In the case of using the impedance given by Melling's eq. to the frequency response, the real diameter $\mathrm{d}_{00}$ should be used in calculation of real part of $\mathrm{z}$ and the equivalent diameter $\mathrm{d}_{0}$ which is calculated by the total hole area should not be used. Where $\mathrm{d}_{0}=\mathrm{d}_{00} \times \sqrt{\mathrm{N}}, \mathrm{N}$ :hole number.

5) In the case of using the impedance given by Melling's eq. to the eigen value analysis, the equivalent diameter $d_{0}$ is allowed to be used in calculation of the imaginary part of $\mathrm{z}$ but the open end correction should be calculated by using $\mathrm{d}_{00}$.

\section{REFERENCES}

[1] Y. Kimura, Z. Yamaguchi, and T. Itaya, Perforated panel type silencer for screw compressors, Kobe Steel Engineering Reports, Vol.59, No.3, 2009, pp.13-16(in Japanese).

[2] K. Tsugihashi, I. Yamagiwa and M. Kikuchi, Research on Numerical Analysis Technique for Sound Fields including Perforated Plate, Kobe Steel Engineering Report, Vol.64, No.2, 2014, pp.90-95(in Japanese)

[3] K. Ishihara, M. Nakaoka and M. Nishioka, Study on a countermeasure for high level sound generated from boiler tube bank duct using walls made of perforated plate (In case of aperture ratio being more than 1\%), Transactions of the J SME (in Japanese), Vol.82, No.841, 2016, DOI: 10.1299/transjsme. 16-00179.

[4] K. Ishihara, Study on a countermeasure for high level sound generated from boiler tube bank duct using walls made of perforated plate (Grasp critical aperture ratio and influence of cavity volume on suppression effect), Transactions of the JSME (in Japanese), Vol.83, No.848, 2017 , DOI: 10.1299/transjsme. 16-00456.

[5] K. Ishihara, S. Kudo, T. Masumoto and M. Mori, Acoustic natural frequency of one dimensional sound field partitioned by perforated plate (comparison between analysis and experiment), Proceedings of the 55th Annular Meeting in Chugoku-Shikoku, 2017, ID: K1114 (in Japanese).

[6] K. Ishihara, S. Kudo, T. Masumoto and M. Mori, Study on acoustic natural frequency and its mode of one dimensional sound field partitioned by perforated plate, Transactions of the JSME (in 
Japanese), Vol.84, No.857, 2018, DOI:10. 1299/transjsme. 17-00365.

[7] K. Ishihara, S. Kudo, Study on acoustic natural frequency of one dimensional sound field partitioned by perforated plate, Transactions of the JSME (in Japanese), Vol.85, No.871, 2019, DOI:10. 1299/transjsme. 18-00305.

[8] T. H. Melling, The acoustic impedance of perforates at medium and high sound pressure levels, Journal of Sound and Vibration, Vol.29, No.1, 1973, pp.1-65.

[9] H. Utsuno and T. Itaya, Experimental study of Sound Absorbing characteristics for perforated panel by Four-microphone measuring technique, Proceedings of JSME Dynamics and Design Conference 2001, on CD-ROM (2001), ID:647(in Japanese).

[10] H. Utsuno, T. Itaya and Z. Yamaguchi, Experimental study on sound absorbing characteristics of perforated panel by transfer matrix method, Journal of Acoustic Society of Japan, Vol.59, No.6, 2003, pp.301-308(in Japanese).

Kunihiko Ishihara was born in 1947 in Kurashiki City, Okayama Prefecture Japan. He received the B.S. degree from Kobe University in 1969. He got a master's degree in Kobe University in 1971 and earned the Ph.D. degree in Engineering from The Osaka University in 1986.

He worked in Kawasaki Heavy Industry Co. Ltd. as an Mechanical Engineer for 33 years. After that he became a Professor of The University of Tokushima in 2004. He had been studying the vibration and noise control, above all he studied the flow induced vibration and noise problems. He has authored or co-authored over 100 technical journal and over 50 conference papers. He is a fellow of JSME (Japan Society of Mechanical Engineers) now. He is a Professor of Tokushima Bunri University. He teaches a mechanical field subjects for students. 